\title{
Co-culture with periodontal ligament stem cells enhances osteogenic gene expression in de-differentiated fat cells
}

\author{
Kallapat Tansriratanawong $\cdot$ Yuichi Tamaki $\cdot$ \\ Hiroshi Ishikawa $\cdot$ Soh Sato
}

Received: 22 January 2014/ Accepted: 3 February 2014/ Published online: 27 February 2014

(C) The Author(s) 2014. This article is published with open access at Springerlink.com

\begin{abstract}
In recent decades, de-differentiated fat cells (DFAT cells) have emerged in regenerative medicine because of their trans-differentiation capability and the fact that their characteristics are similar to bone marrow mesenchymal stem cells. Even so, there is no evidence to support the osteogenic induction using DFAT cells in periodontal regeneration and also the co-culture system. Consequently, this study sought to evaluate the DFAT cells co-culture with periodontal ligament stem cells (PDLSCs) in vitro in terms of gene expression by comparing runtrelated transcription factor 2 (RUNX2) and Peroxisome proliferator-activated receptor gamma 2 (PPAR $\gamma 2)$ genes. We isolated DFAT cells from mature adipocytes and compared proliferation with PDLSCs. After co-culture with PDLSCs, we analyzed transcriptional activity implying by DNA methylation in all adipogenic gene promoters using combined bisulfite restriction analysis. We compared
\end{abstract}

K. Tansriratanawong $(\bowtie) \cdot H$. Ishikawa

Department of NDU Life Sciences, Nippon Dental University School of Life Dentistry at Tokyo, 1-9-20 Fujimi, Chiyoda-ku, Tokyo 102-8159, Japan

e-mail: tong_dent@hotmail.com

K. Tansriratanawong $\cdot S$. Sato

Department of Periodontology, Nippon Dental University School of Life Dentistry at Niigata, 1-8 Hamaura-cho, Chuo-ku,

Niigata 951-1500, Japan

K. Tansriratanawong

Department of Oral Medicine and Periodontology, Faculty of Dentistry, Mahidol University, 6 Yothi Street Rajthevi,

Bangkok 10400, Thailand

Y. Tamaki

Department of Developmental and Regenerative Dentistry, Nippon Dental University School of Life Dentistry at Tokyo, 1-9-20 Fujimi, Chiyoda-ku, Tokyo 102-8159, Japan gene expression in RUNX2 gene with the PPAR $\gamma 2$ gene using quantitative RT-PCR. After being sub-cultured, DFAT cells demonstrated morphology similar to fibroblastlike cells. At the same time, PDLSCs established all stem cell characteristics. Interestingly, the co-culture system attenuated proliferation while enhancing osteogenic gene expression in RUNX2 gene. Using the co-culture system, DFAT cells could trans-differentiate into osteogenic lineage enhancing, but conversely, their adipogenic characteristic diminished. Therefore, DFAT cells and the coculture system might be a novel cell-based therapy for promoting osteogenic differentiation in periodontal regeneration.

Key words Co-culture $\cdot$ PDLSCs - DFAT cells · Osteogenesis · DNA methylation

\section{Introduction}

The ultimate goal of periodontal therapy is periodontal regeneration, which is defined as the establishment and reconstruction of new periodontium into functional architectures using lost or injured tissues [1,2]. While we used several procedures to achieve regeneration, stem cells produced the best outcomes. Dental stem cells are categorized in cell-based procedures for tissue engineering. They derived from dental organs, including dental pulp, periodontal ligament, root apical papilla, and dental follicle, which possess a high potential for use in regenerative medicine [3-6]. They share characteristics similar to mesenchymal stem cells (MSCs) in terms of their colonyforming efficiency, proliferation, and multi-lineage differentiation. Among these, periodontal ligament stem cells (PDLSCs) have shown potential in osteogenic 
differentiation for periodontal regeneration and have also exhibited multi-lineage differentiation into adipogenic, chondrogenic, and neurogenic lineage [7-9]. It was possible to isolate PDLSCs from the heterogeneous population in periodontal ligaments, which contained varieties of progenitor cells and differentiating cells, using single colony cloning and stem cell markers sorting. They expressed the surface stem cell antigen markers, which included CD44, CD73, CD90, CD105, CD146, and Stro-1 [5, 10, 11].

Beyond dental stem cells, alternative sources for cellbased therapy for periodontal regeneration have gained more attention in somatic stem cells. Recently, de-differentiated fat cells (DFAT cells) emerged as a possible alternative cell source for regeneration. Subcutaneous fat tissue can be harvested easily and sufficiently obtained in appropriate amount for regenerative defects as compared to PDLSCs. Successfully isolating them using the ceiling method demonstrated characteristics similar to bone marrow mesenchymal stem cells (BMMSCs) and adiposederived stem cells (ASCs) [12-15]. Moreover, much evidence showed that DFAT cells could trans-differentiate into other cell types, such as cardiocytes [16], smooth muscle-like cells [17], and urethral sphincter cells [18]. While the role of DFAT cells for periodontal regeneration and their potential for osteogenic differentiation nevertheless remains unclear, a recent study hypothesized that DFAT cells might provide the trans-differentiation property for osteogenic differentiation for periodontal regeneration.

A co-culture system was used for cell culture improvement, mechanism investigation, and cell-cell interaction simulation, taking place between endothelial cells with MSCs, including in PDLSCs. A previous study demonstrated the signaling pathway of COX-2/PGE2/VEGE under the hypoxia condition up-regulating in osteogenic differentiation of PDLSCs after co-cultured with endothelial cells [19]. Moreover, a co-culture of endothelial cells with DFAT cells appeared to reverse the stemness characteristic and morphology similar to immature adipocytes [20]. Recently, a co-culture with MSCs performed in oral cells type provided immature features by expressing stem cell-associated genes [21]. Thus, we supposed that coculture system with PDLSCs might not only simulate the periodontal environment but also provide the enhancement in osteogenic induction for DFAT cells.

Osteogenic and adipogenic lineages are recognized as the contrary lineage in MSCs differentiation [22]. Particularly, in runt-related transcription factor 2 (RUNX2) and Peroxisome proliferator-activated receptor gamma 2 (PPAR $\gamma 2)$, they are proposed for flavor regulation in their osteogenic or adipogenic differentiation. We used DFAT cells, which are representative of the adipogenic lineage, and focused on whether DFAT cells might trans-differentiate into osteogenic differentiation and up-regulate the osteogenic gene expression.

We hypothesized that a co-culture of DFAT cells with PDLSCs might simulate the periodontal environment in vitro and enhance the osteogenic differentiation function for periodontal regeneration. This study aimed to evaluate the osteogenic gene expression of DFAT cells after being co-cultured with PDLSCs by detecting the RUNX2 gene expression level and comparing its effect to the PPAR $\gamma 2$ gene.

\section{Materials and methods}

\section{Isolation and culture of DFAT cells}

The protocol was approved by the ethics committees of Nippon Dental University (NDU-T2011-32). Subcutaneous adipose tissues were obtained from three healthy female subjects (58-85 years), who were given their written informed consents. Adipose tissues were isolated in accordance with ceiling method procedure [12]. Tissues were digested with warmed $3 \mathrm{mg} / \mathrm{mL}$ collagenase type I (Sigma, St Louis, MO) and $4 \mathrm{mg} / \mathrm{mL}$ dispase (Sanko Pure Chemical Ltd., Tokyo, Japan) at $37^{\circ} \mathrm{C}$ for $1 \mathrm{~h}$ and subsequently centrifuged at $300 \times g$ for $15 \mathrm{~min}$. Mature fat cells at the uppermost portion were collected following by incubating with erythrocyte lysis buffer at $4{ }^{\circ} \mathrm{C}$ for $15 \mathrm{~min}$. Cell suspensions were then filtered through $70-\mu \mathrm{m}$ nylon cell strainers (Falcon, BD Labware, Franklin Lakes, NJ) and seeded approximately $1 \times 10^{5}$ cells in each $25-\mathrm{cm}^{2}$ culture flask (NUNC, Roskilde, Denmark), which completely filled with growth medium (GM). Dulbecco's modified Eagle's medium/Ham's nutrient mixture F12 (Gibco BRL, Carlsbad, CA) supplemented with $15 \%$ fetal bovine serum (Gibco), $2 \mathrm{mM}$ glutamine (GlutaMAX I, Invitrogen, Carlsbad, CA), $50 \mathrm{U} / \mathrm{mL}$ penicillin and $50 \mu \mathrm{g} / \mathrm{mL}$ streptomycin (Gibco BRL) were used as GM. Mature fat cells floated and attached to the upper surface of the flask. Then, flask was inverted with reduction the medium at 7-10 days. For cell morphology investigation, DFAT cells were rinsed with phosphate buffer saline (PBS) followed by fixed in $10 \%$ formalin solution, and stained with Oil Red O (Wako). On the other hand, DFAT cells culture, which reached to confluence, were then sub-cultured by adding $0.1 \%$ trypsin and $0.02 \%$ ethylenediaminetetraacetic acid (EDTA)/PBS and split at 1:3 dilution in fresh medium.

Isolation and culture of PDLSCs and BMMSCs

The periodontal ligaments at middle one-third of the impacted or premolar tooth roots from three healthy female 
subjects (17-25 years) were harvested and cut into small pieces following digested by enzyme. Isolation protocol was followed as described earlier [7]. Cell suspension was filtered through $70 \mu \mathrm{m}$ nylon cell strainer and then, centrifugation was performed at $300 \times g$ for $15 \mathrm{~min}$. Cells were retrieved in GM and approximately $1 \times 10^{4}$ cells were seeded in each 100-mm dish (Nunc) as primary culture. For BMMSCs, three cell lines from passage (P) 3 were used as a control of MSCs [7].

Population doubling time (PDT)

For determination of proliferative function, DFAT cells and PDLSCs were seeded at cell density of $1 \times 10^{4}$ cells into 35-mm dish (Falcon). The numbers of cells were counted in triplicate every 2 days for 2 weeks. PDT was calculated by PDT software [40].

Flow cytometric analysis

PDLSCs from P3 were harvested by trypsinization and split approximately $5 \times 10^{5}$ cells per tube. Mouse monoclonal anti-human antibodies conjugated with fluorescein isothiocyanate-conjugated (FITC) and phycoerythrin (PE) were performed as follow: anti-CD-90-PE, anti-CD105-PE, anti-CD106-PE, and isotype control using immunoglobulin $\mathrm{G}$ (all from BD Biosciences, San Jose, CA); anti-CD-34FITC, and anti-CD-44-FITC (Beckman coulter). Each aliquot was incubated in the dark at $4{ }^{\circ} \mathrm{C}$ for $20 \mathrm{~min}$. Cell pellets were washed with PBS and resuspended in $1 \%$ BSA/PBS. Flow cytometric analysis was performed in triplicate and determined in quantitative data using Guava Express Plus version 5.3 software (Guava Technology).

\section{Multilineage differentiation}

PDLSCs were plated at density $1 \times 10^{4}$ cells per well in 6-well plate. Once PDLSCs reached to the confluence, each differentiation medium was then substituted. Osteogenic differentiation was supplemented with $100 \mathrm{nM}$ dexamethasone, $50 \mu \mathrm{M}$ ascorbic acid, and $10 \mathrm{mM} \beta$-glycerophosphate. Adipogenic differentiation was supplemented with $1 \mu \mathrm{M}$ dexamethasone, $0.5 \mathrm{mM}$ isobutylmethylxanthine (IBMX), and $100 \mu \mathrm{M}$ indomethacin. Chondrogenic differentiation was supplemented with $10 \mathrm{ng} / \mathrm{mL}$ transforming growth factor beta-1 (TGF- $\beta 1$ ), $100 \mathrm{nM}$ dexamethasone, $37.5 \mu \mathrm{g} / \mathrm{mL}$ ascorbic acid, $1 \%$ insulintransferrin-selenium (ITS), and $1 \mathrm{mM}$ sodium pyruvate. All lineage differentiations were cultured for 3 weeks subsequently by fixation with $10 \%$ formalin solution and stained as follows: osteogenic differentiation was stained by $1 \%$ Alizarin Red (Certistain ${ }^{\circledR}$, Darmstadt, Germany) at $\mathrm{pH} 4.2$ for $30 \mathrm{~min}$, adipogenic differentiation was stained by Oil Red $\mathrm{O}$, and chondrogenic differentiation was stained by $0.1 \%$ Toluidine Blue (Muto Pure Chemical, Japan), respectively.

Reverse transcriptase polymerase chain reaction (RT-PCR)

Multilineage differentiation was confirmed genes expression by RT-PCR. Total RNA was extracted using RNeasy Mini kit (Qiagen, Hilden, Germany) according to the manufacturer's protocol and determined quantity of RNA by $260 / 280 \mathrm{~nm}$ absorbance. cDNA was synthesized from $1 \mu \mathrm{g}$ of RNA using the High Capacity cDNA synthesis kit (Applied Biosystems, Carlsbad, CA). The PCR Supermix Platinum kit (Invitrogen) was used for amplification following by condition of preincubation at $94{ }^{\circ} \mathrm{C}$ for $2 \mathrm{~min}$, then performed by 35 cycles of denaturation at $94{ }^{\circ} \mathrm{C}$ for $30 \mathrm{~s}$; primer annealing at $52-60{ }^{\circ} \mathrm{C}$ for $30 \mathrm{~s}$ and extension step at $72{ }^{\circ} \mathrm{C}$ for $1 \mathrm{~min}$. Finally, a post extension step was done at $72{ }^{\circ} \mathrm{C}$ for $7 \mathrm{~min}$. PCR products were electrophoresed using $2 \%$ agarose gel being stained with $0.5 \mu \mathrm{g} / \mathrm{mL}$ ethidium bromide (EtBr). RT-PCR primers are listed in the Table 1. GAPDH was used as an endogenous control.

\section{Co-culture system}

For construction of cell-cell interactive environment, DFAT cells and PDLSCs from P3 were used. DFAT cells were plated at the density $1 \times 10^{4}$ cells/well in 6 -well plate (Sumilon, Sumitomo, Japan). On the contrary, PDLSCs were plated $1 \times 10^{3}$ cells/well in the $0.4 \mu \mathrm{m}$ pore size of 6-transwell insertion (Falcon). Co-culture DFAT cells with PDLSCs were extended for 2 weeks followed by DNA extraction and methylation analysis. On the other hand, for determining osteogenic gene expression, co-culture was continuously cultured for further 2 weeks replacing by osteogenic differentiation medium as described earlier in multilineage differentiation. Non-co-culture group was defined DFAT cells culture without PDLSCs.

\section{DNA extraction and bisulfite conversion}

Genomic DNA was extracted from co-culture, non-coculture, and BMMSCs group using the DNeasy ${ }^{\circledR}$ Blood \& Tissue kit (Qiagen). Cells were digested by lysis buffer from manufacturer and isolated DNA aliquot. Then, bisulfite modification was performed to DNA using the EpiTect ${ }^{\circledR}$ bisulfite kit (Qiagen). In brief, $1 \mu \mathrm{g}$ of DNA was mixed with the bisulfite mixture and carried out thermal cycler approximately $5 \mathrm{~h}$. PCR was used for amplifying bisulfite modified DNA as follows: preincubation at $94{ }^{\circ} \mathrm{C}$ for $2 \mathrm{~min}$, then performed by 40 cycles of denaturation at $94{ }^{\circ} \mathrm{C}$ for $1 \mathrm{~min}$; primer annealing at $54-57^{\circ} \mathrm{C}$ for $1 \mathrm{~min}$, 


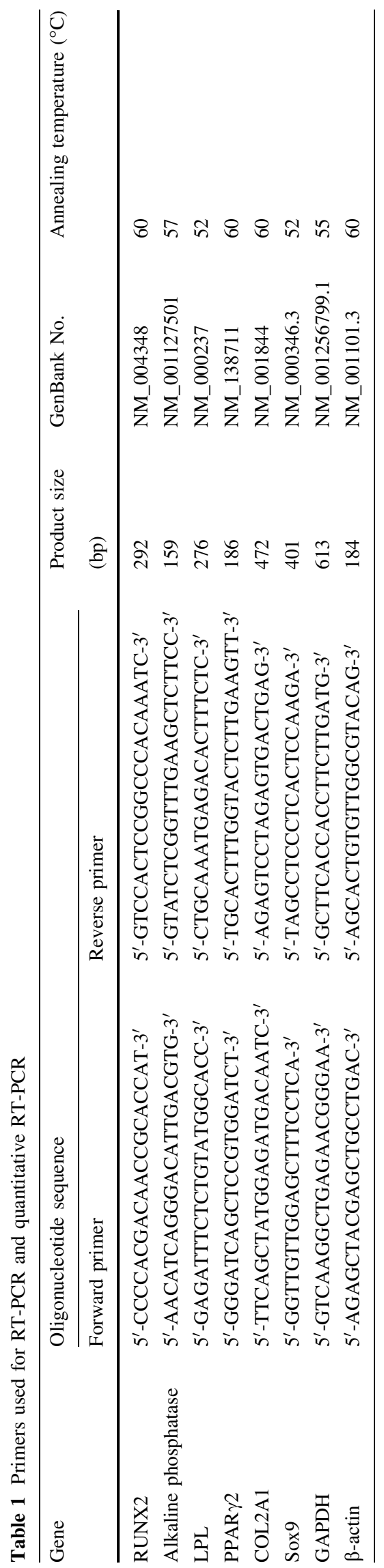

extension step at $65{ }^{\circ} \mathrm{C}$ for $1 \mathrm{~min}$, and post extension step at $65{ }^{\circ} \mathrm{C}$ for $7 \mathrm{~min}$ using bisulfite primers sets for CCAAT/ enhancer binding protein alpha $(\mathrm{C} / \mathrm{EBP} \alpha)$, Fatty acid binding protein 4 (FABP4), Lipoprotein lipase (LPL), PPAR $\gamma 2$, and RUNX2 gene promoters as shown in Table 2 .

Combined bisulfite restriction analysis (COBRA)

PCR products of bisulfite modified DNA from all groups were digested with $20 \mathrm{U}$ of restriction enzymes overnight, which were specific in the restriction sites by $H p y C H 4 I V$ (ACGT) for C/EBP $\alpha$, LPL, PPAR $\gamma 2$ and Taq I (TCGA) for FABP4 and RUNX2 gene (New England Biolabs, Ipswich, MA, USA). The digested PCR products were electrophoresed using $2 \%$ agarose gel and being stained with $0.5 \mu \mathrm{g} / \mathrm{mL}$ of EtBr. Each gene fragment length was shown as follows: $\mathrm{C} / \mathrm{EBP} \alpha$, the amplicons provided 171,249 , and 420 base pair (bp): FABP4, the amplicons provided 56, 85, 141, 272, 357, and 413 bp: LPL, the amplicons provided 121, 164, 172, 285, 336, and 457 bp: PPAR $\gamma 2$, the amplicons provided 62 , 181, 237, 299, 418, and 480 bp: and RUNX2, the amplicons provided 103, 261, and 364 bp. MultiGauge V3.0 software (Fujifilm, Japan) was analyzed each band intensity and methylation in percentage, which were calculated by following formula: methylation percentage $=100 \times$ digested fragments/undigested fragments + digested fragments.

\section{Real-time PCR and semi-quantitative RT-PCR}

Osteogenic differentiation potential from co-culture and non-co-culture group was compared in RUNX2 and PPAR $\gamma 2$ gene. The comparison of relative gene expression from RUNX2 and PPAR $\gamma 2$ gene was indicated by Power SYBR $^{\circledR}$ Green PCR Master Mix (Life Technologies, UK). The $\beta$-actin was used as an endogenous control. Real-time PCR primers are included in list of Table 1. For real-time PCR reaction, $500 \mathrm{ng}$ of cDNA, $5 \mu \mathrm{M}$ of each forward and reverse primer, $10 \mu \mathrm{L}$ of SYBR Green, and distilled water were mixed in 96-well plate. The condition was performed preincubation at $95{ }^{\circ} \mathrm{C}$ for $10 \mathrm{~min}$, followed by 40 cycles of denaturation at $95{ }^{\circ} \mathrm{C}$ for $15 \mathrm{~s}$; primer annealing at $60{ }^{\circ} \mathrm{C}$ for $1 \mathrm{~min}$ and extension step at $95{ }^{\circ} \mathrm{C}$ for $15 \mathrm{~s}$. Data were analyzed by StepOne ${ }^{\mathrm{TM}}$ software version 2.1. For semi-quantitative RT-PCR, serial concentrations were measured the band density using MultiGauge software. Relative band densities were calculated by normalization to GAPDH, which was used as an endogenous control.

Statistical analysis

Data were reported as mean \pm SD. Independent sample $t$ test was used for analysis two-group comparison, whereas 


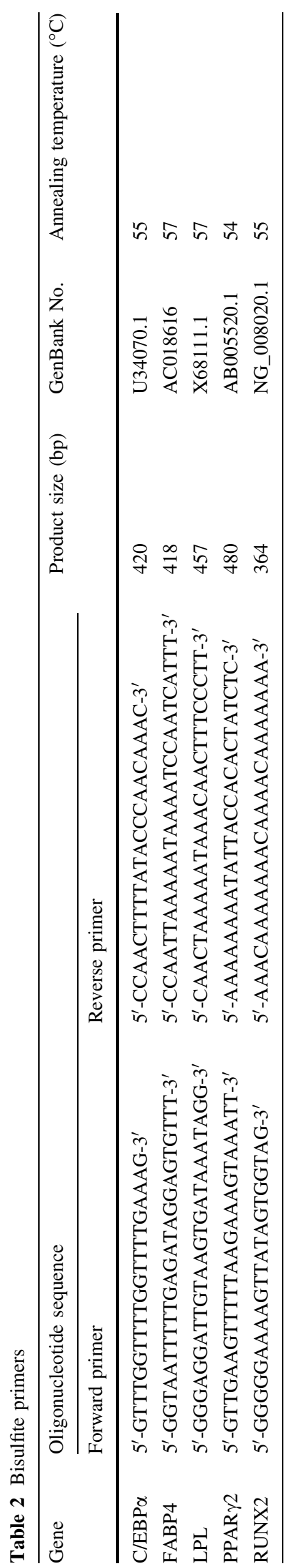

One-way ANOVA or Kruskal-Wallis test was used for the intergroup comparison. Differences at $P<0.05$ were considered to be statistically significant. All experiments were performed in triplicate and were repeated with isolated cells from different subjects.

\section{Results}

DFAT cells can de-differentiate from mature fat cells to fibroblast-like cells

DFAT cells were successfully isolated by the ceiling method, which could de-differentiate and proliferate from mature fat cells to fibroblast-like cells. In this study, DFAT cells displayed high proliferation potential after sub-cultured of primary culture. However, morphology gradually changed to all polyhedral shape in P7 (Fig. 1a, b). From day 7 to 10 in primary culture, DFAT cells demonstrated morphologic heterogeneity including fibroblast-like cells, polyhedral cells, and cell containing lipid droplets, which positively stained by Oil Red O (Fig. 1c, d). On the other hand, PDLSCs appeared in all fibroblast-like cells and actively expanded (Fig. 1e).

PDLSCs exhibit higher proliferation than DFAT cells

PDLSCs and DFAT cells were compared the proliferative function in PDT for 2 weeks. PDLSCs exhibited approximately 2 times shorter in PDT (2.62) when compared with DFAT cells (5.04) (Fig. 1f).

Stem cell characterizations of PDLSCs

To confirm stem cell characteristics of PDLSCs, immunophenotypes by flow cytometry, and multilineage differentiation were performed. PDLSCs demonstrated cell surface antigen markers of MSCs (mean percentage $\pm \mathrm{SD}, n=3)$, including CD90 (99.93 \pm 0.1$)$, CD105 $(85.66 \pm 0.06)$, and adherence cell marker, CD44 $(98.78 \pm 1.73)$; in contrast, CD34, which was a hematopoietic stem cell marker, was negatively found $(0.26 \pm 0.13)$. CD106, which is the vascular adhesion molecule (VCAM-1), was detected from PDLSCs approximately $8.15 \pm 0.36$. All surface antigen markers were compared to negative control using isotype $\mathrm{IgG}$ (Fig. 2a). PDLSCs were induced in three different differentiated media for multilineage differentiation, including osteogenic (Alizarin Red staining), adipogenic (Oil Red O staining), and chondrogenic (Toluidine Blue staining) differentiation for 3 weeks. All lineages were confirmed genes expression by RT-PCR (Fig. 2b). 
Fig. 1 Morphologies and population doubling time comparison of DFAT cells and PDLSCs. a, b DFAT cells from passage 1 (P1) mostly demonstrated in fibroblast-like cells but gradually flatten and provided polyhedral morphology when cultured to the passage 7 (P7). DFAT cells, which were isolated from 7 to 10 days of primary culture, were positive stained lipid droplets by Oil Red O (c, d). PDLSCs exhibited fibroblastlike cells homogenously (e). Population doubling time (PDT) comparing between DFAT cells and PDLSCs was analyzed every 2 days for 2 weeks. PDLSCs provided shorter PDT, which implied as higher proliferation potential (f). Scale bar without character indicated $100 \mu \mathrm{m}$
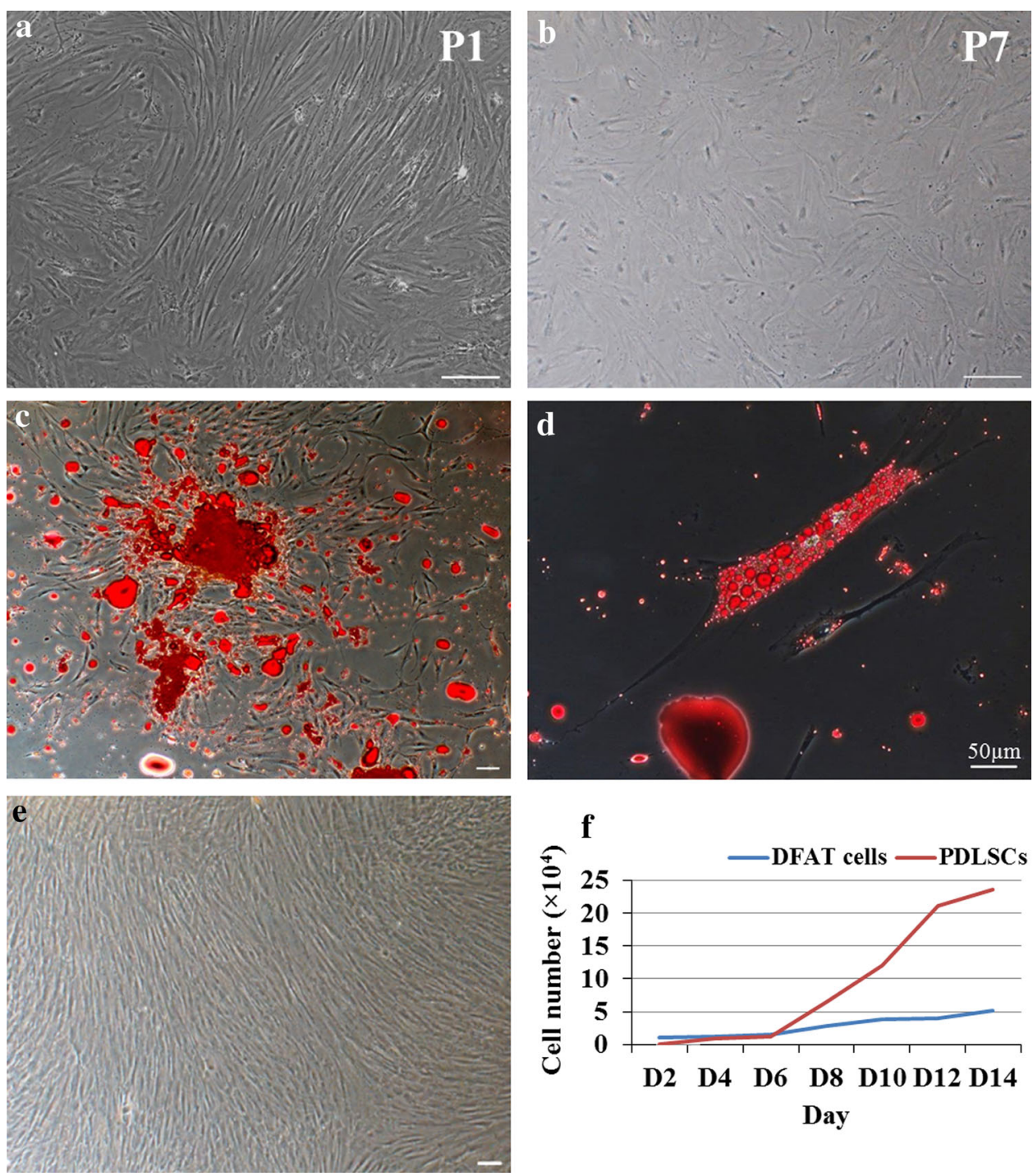

Co-culture system up-regulates methylation status in all adipogenic gene promoters but down-regulates in osteogenic RUNX2 gene promoter

DFAT cells were co-cultured with PDLSCs in 6-transwell plates for 2 weeks. Co-culture group demonstrated sparse cell distribution that mostly contained polyhedral morphology, but non-co-culture group and PDLSCs demonstrated fibroblastlike cells (Fig. 3a). DNA methylations from non-co-culture and co-culture group were analyzed in $\mathrm{C} / \mathrm{EBP} \alpha, \mathrm{FABP} 4, \mathrm{LPL}$, and PPAR $\gamma 2$ genes using specific restriction enzymes digested at the cytocine phosphate guanine $(\mathrm{CpG})$ sites (Fig. 4a). Digested and un-digested DNA fragments from all groups were verified the band intensities (Fig. 4b). All were compared DNA methylation status in mean of percentage \pm SD. $\mathrm{C} / \mathrm{EBP} \alpha$ and LPL gene demonstrated statistically significant difference in methylation profiles after co-cultured, which increased from $47.12 \pm 0.54$ to $51.87 \pm 0.58$, and
$66.9 \pm 2.27$ to $77.29 \pm 0.11(P<0.01,0.05)$, respectively. All genes from both groups except in FABP4 gene have shown statistically significant difference when compared with BMMSCs $(\mathrm{P}<0.05)$ (Fig. 4c; Table 3). On the contrary, DNA methylation percentage of RUNX2 gene significantly reduced after co-cultured, which displayed $57.41 \pm 2.16$, $47.82 \pm 2.9$, and $47.04 \pm 4$ in non-co-culture, co-culture, and BMMSCs, respectively (Fig. 4d).

Osteogenic differentiation potential of DFAT cells is enhanced after PDLSCs co-culture

After 2 weeks osteogenic induction, gene expression levels of RUNX2 and PPAR $\gamma 2$ gene were determined using realtime PCR normalizing by $\beta$-actin. RUNX 2 gene expression significantly enhanced upon using co-culture system. It provided RUNX2 gene up-regulation higher than control and non-co-culture group $(P<0.05)$ (Fig. 5a). On the 


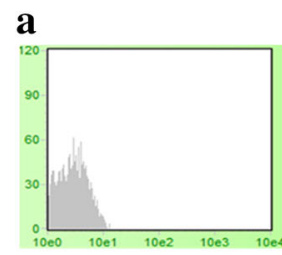

Negative control (IgG)

b

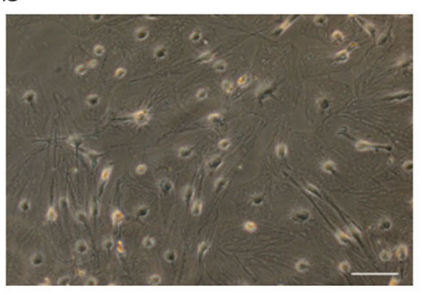

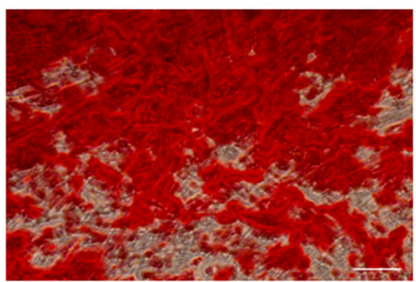

Osteogenic differentiation
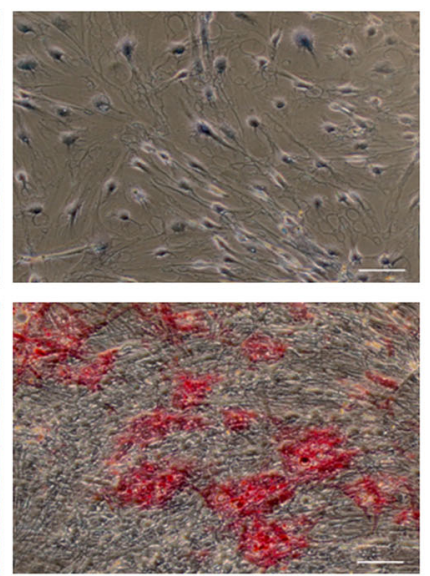

Adipogenic differentiation

CD 34

CD 44

CD 90

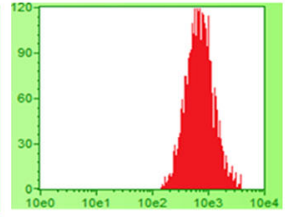

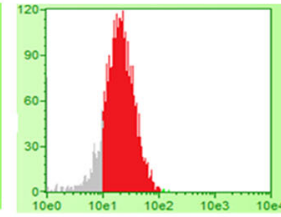

CD 105

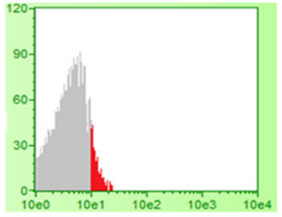

CD 106
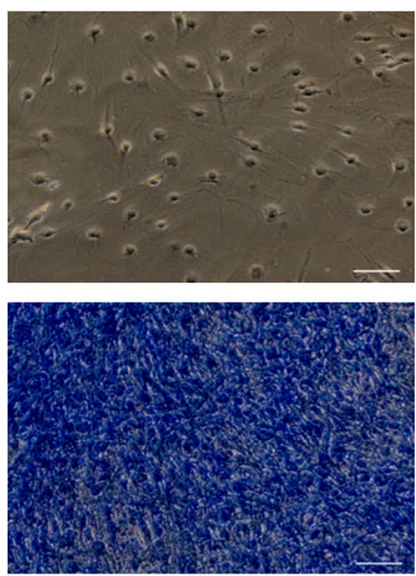

Chondrogenic differentiation

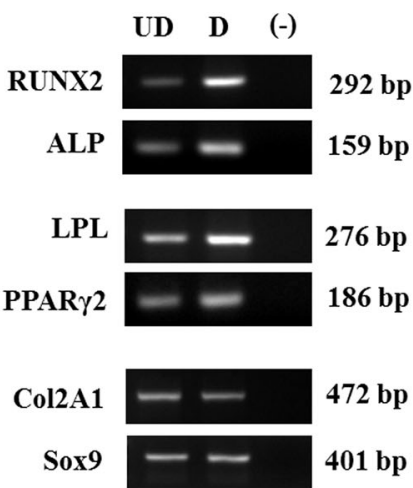

GAPDH

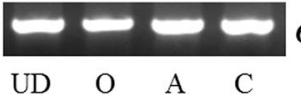

Fig. 2 Stem cells characterizations of PDLSCs. a Flow cytometric analysis was performed for detecting immunophenotypes, which displayed all mesenchymal stem cell markers: CD44, CD90, CD105 but negatively shown the vascular cell markers: CD34 and CD106. Immunoglobulin $\mathrm{G}$ was used as a negative control, which demonstrated in all grey filled. Positive cell expressions were present by red filled. b PDLSCs were successfully induced into osteogenic (Alizarin Red

contrary, PPAR $\gamma 2$ gene expression level demonstrated attenuation profile in co-culture group. Unfortunately, data did not provide statistically significant difference when compared with non-co-culture group (Fig. 5b). For semiquantitative RT-PCR, the relative band densities were evaluated with normalization by GAPDH in serial RNA concentration. Both genes also demonstrated the similar patterns to real-time PCR. RUNX2 gene in co-culture group was gradually increased by concentration (Fig. 5c). However, for PPAR $\gamma 2$ gene, co-culture group was expressed lower than non-co-culture group at 0.5 and $1.5 \mu \mathrm{g}$ of RNA concentration (Fig. 5d).

\section{Discussion}

Using cell-based therapy in periodontal regeneration, particularly from tooth-derived stem cells, is widely understood and has proven extremely potent for osteogenic staining), adipogenic (Oil Red O staining), and chondrogenic (Toluidine Blue staining) differentiation, which were confirmed gene expression of each lineage by RT-PCR. GAPDH was used as endogenous control. UD means undifferentiated PDLSCs, (-) means negative control. O means osteogenic-differentiated PDLSCs. A means adipogenic-differentiated PDLSCs. C means chondrogenic-differentiated PDLSCs. bp means base pairs. Scale bar indicated $100 \mu \mathrm{m}$

differentiation. Even so, insufficient stem cell harvesting and high heterogeneity remain the limitations of PDLSCs. Consequently, other somatic stem cells have gained more attention for periodontal regeneration [3-6, 23-25]. Although DFAT cells derived from mature adipocytes are not stem cells, they provided homogeneity and a high expansion capability similar to that of other MSCs, such as BMMSCs and ASCs [12, 15]. Since they were an easily manipulated and abundant source, using DFAT cells in periodontal regeneration might be a novel source for cellbased therapy.

Our study first demonstrated the transcriptional and proliferative function, which was implied by DNA methylation profiles. All adipogenic genes, including $\mathrm{C} / \mathrm{EBP} \alpha$, FABP4, LPL, and PPAR $\gamma 2$, increased DNA methylation in co-culture when compared with non-co-culture and control groups. Co-culture groups of all genes were indicated as hypermethylation, which was defined by methylation status more than $50 \%$. The hypermethylation status could down- 

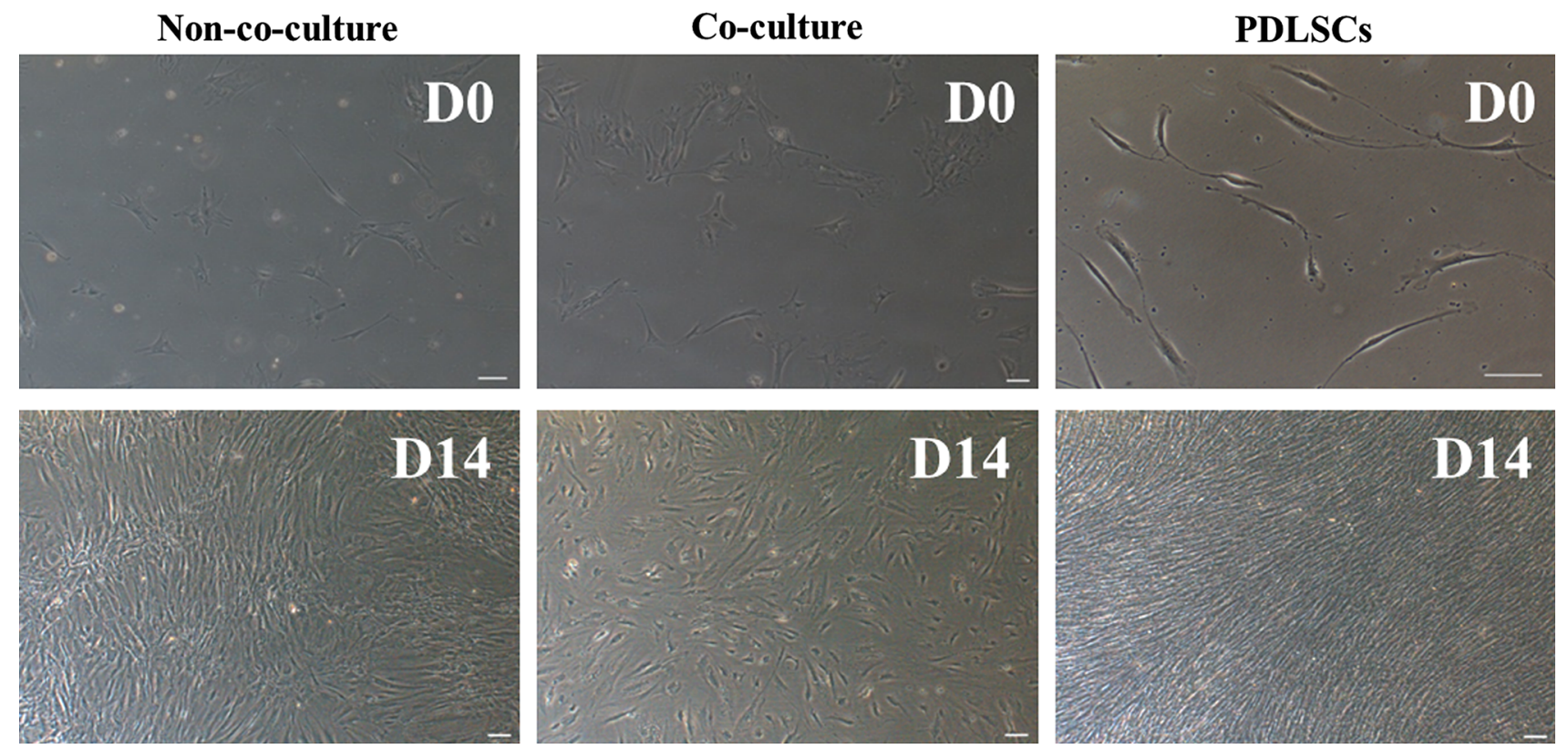

Fig. 3 Cell morphology and distribution after co-cultured. Co-culture system was performed for 2 weeks followed by identifying cellular morphology. DFAT cells from co-culture group have dispersedly in

regulate the transcriptional function, which resulted in silencing of the gene promoter and diminish in gene expression [26-28]. Our findings were consistent with a previous report that demonstrated an attenuated proliferative function in MSCs co-culture with three oral cells types but provided in low mitogenesis in the BrdU level than other gene expression activity [21]. On the other hand, our findings demonstrated that the methylation of RUNX2 gene established a converse effect to adipogenic genes in the coculture group. The suggestion, therefore, was that the coculture system might enhance the transcriptional function of RUNX2 gene.

In osteogenic differentiation, we examined RUNX2 and $\operatorname{PPAR} \gamma 2$, which were gene expressions of osteogenic and adipogenic lineage. We compared these using real-time PCR and semi-quantitative RT-PCR after induction of an osteogenic differentiation medium. The DFAT cells coculture displayed the greatest RUNX2 gene expression when compared with non-co-culture and control groups. Meanwhile, the PPAR $\gamma 2$ gene expression in the co-culture group demonstrated the lowest compared with the others. These indicated the linkage between the two contrary lineages in RUNX2 and PPAR $\gamma$ comparisons. Once RUNX2 is the preferable up-regulation, it drives the osteogenic differentiation and inhibits the PPAR $\gamma$. The proposed manipulating this linkage using the transcriptional co-activator with a PDZ-binding motif or TAZ, which is the transcriptional co-activator used in RUNX2 for osteocalcin expression and PPAR $\gamma$ inhibition. TAZ plays the crucial role in binding with the 14-3-3 protein cell distribution and shown more polyhedral shape comparing to nonco-culture group. Scale bar indicated $100 \mu \mathrm{m}$

binding domain and the Pro-Pro-X-Tyr (PPXY) motif, which contains protein through the WW domain. Since both the RUNX2 and the PPAR $\gamma$ contain the PPXY domain, TAZ can interact with either RUNX2 or PPAR $\gamma$ to activate osteogenic differentiation while inhibiting $\operatorname{PPAR} \gamma$ [22, 29-31]. Previous study demonstrated that transfected murine myoblast $\mathrm{C} 2 \mathrm{C} 12$ cells by siRNA against TAZ isoform could inhibit the causal chain of osteoblastic differentiation via BMP-2 and osteocalcin gene expression by RUNX2 regulation. It was supposed that interaction of TAZ with RUNX2 effectively stimulated the osteocalcin gene promoter activity, a late marker of osteoblastic regeneration. On the other hand, TAZ binding to PPAR $\gamma$ could inhibit transcription from the aP2 gene promoter, which result in adipogenic differentiation down-regulation [22]. From these findings, we supposed that osteogenic and adipogenic lineages interacted oppositely due to a coordinating factor like TAZ.

RUNX2 and PPAR $\gamma$ are the pivotal transcriptional factors that can modulate MSCs into differentiating to the osteogenic or adipogenic lineage [32-35]. RUNX2 plays an essential role in osteoblastic differentiation and controls downstream target genes such as osteocalcin [36, 37]. It is possible to switch the MSCs for lineage differentiation depending upon the flavor factors and appropriate environment [22]. On the contrary, PPAR $\gamma$ is the key regulatory factor for adipogenic differentiation. A recent report has proposed a possible association between osteogenic and adipogenic differentiation that might be controlled via the signaling pathway of BMP4 and TNF- $\alpha$. The PPAR $\gamma$ 

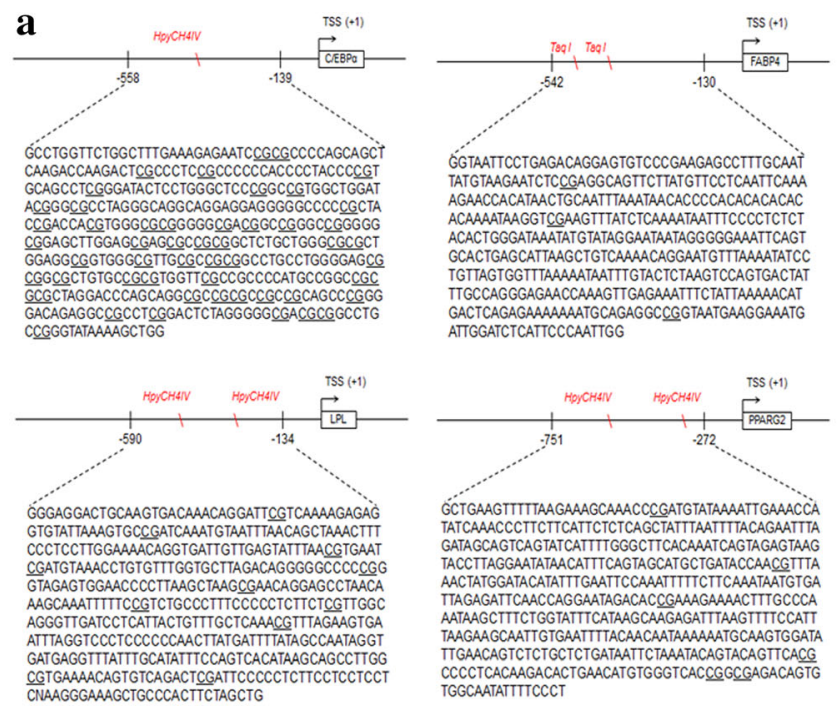

c

$\square$ Non-co-culture $\square$ Co-culture $\square$ BMMSCs

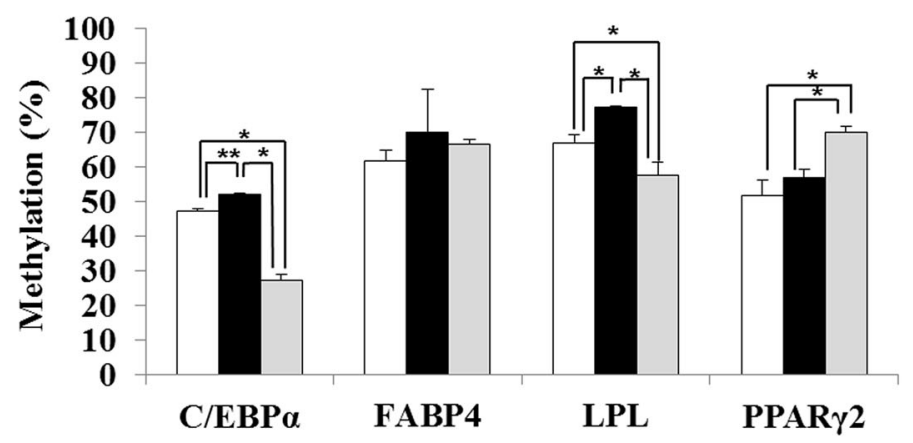

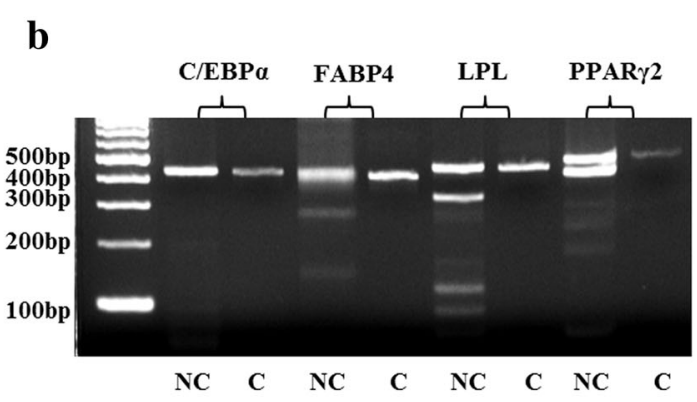

d

RUNX2

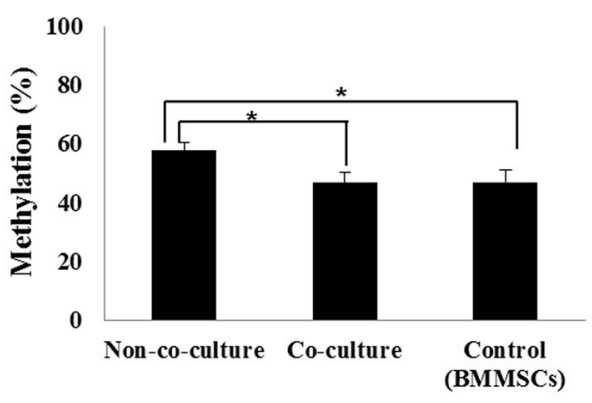

Fig. 4 DNA methylation profiles of four adipogenic genes and RUNX2 gene by COBRA technique. (a, b) After co-cultured for 2 weeks, DNA methylation analysis of four adipogenic genes was analyzed by COBRA technique. Each PCR product was digested by restriction enzymes; HpyCH4IV (ACGT) for C/EBP $\alpha$, LPL, PPAR $\gamma 2$ and Taq I (TCGA) for FABP4 and providing fragments as follows: $\mathrm{C} / \mathrm{EBP} \alpha$ gene $(171,249$, and $420 \mathrm{bp}), \mathrm{FABP} 4$ gene $(56,85,141,272$, 357 , and $413 \mathrm{bp}$ ), LPL gene (121, 164, 172, 285, 336, and $457 \mathrm{bp}$ ), and PPAR $\gamma 2$ gene $(62,181,237,299,418$, and 480 bp). c Co-culture

Table 3 DNA methylation in percentage of four adipogenic genes

\begin{tabular}{lcll}
\hline Gene & Non-co-culture & Co-culture & BMMSCs \\
\hline C/EBP $\alpha$ & $47.12 \pm 0.54$ & $51.87 \pm 0.58$ & $27.17 \pm 1.57$ \\
FABP4 & $61.71 \pm 2.9$ & $70.03 \pm 12.41$ & $66.53 \pm 1.43$ \\
LPL & $66.9 \pm 2.27$ & $77.29 \pm 0.11$ & $57.57 \pm 3.62$ \\
PPAR $\gamma 2$ & $51.75 \pm 4.23$ & $56.77 \pm 2.36$ & $69.97 \pm 1.83$ \\
\hline
\end{tabular}

Mean \pm SD

can suppress the effects of the BMP-type 2 receptor and the smad1/5/8 signaling thus resulting in adipogenic differentiation. At the same time, BMP4 and TNF- $\alpha$ could also down-regulate reversely to the PPAR $\gamma$ via the SAPK/JNK/ group demonstrated higher in methylation status in all adipogenic genes when compared with non-co-culture group, which implied for retardation in transcriptional activity. d RUNX2 gene has adversely demonstrated the methylation profile by showing lower methylation status after performed co-culture. NC means non-co-culture. $C$ means co-culture, $b p$ means base pairs. Scale bar indicated $100 \mu \mathrm{m}$. Astrerisk means that comparison was statistically significant difference at $P<0.05$, double asterisk means that comparison was statistically significant difference at $P<0.01$

$\mathrm{NF} \kappa \mathrm{B} / \mathrm{Stat}$ signaling pathway, which provides the up-regulation of RUNX2 and osteogenesis [38].

The utility of the co-culture system has rarely been proposed regarding DFAT cells. A previous report demonstrated mature fat cells with endothelial cells co-culture. The histological feature of DFAT cells after co-culture demonstrated that the pre-adipocyte-like cells occurred in conjunction with DFAT cells and endothelial cells and generally expressed Flk-1, which was the endothelial cell marker. Moreover, DFAT cells also induced the endothelial cells by trans-differentiating into preadipocyte-like cells [20]. In terms of its osteogenic differentiation potential, the co-culture system could serve as an inductive process for PDLSCs when performed using endothelial cells. Several 

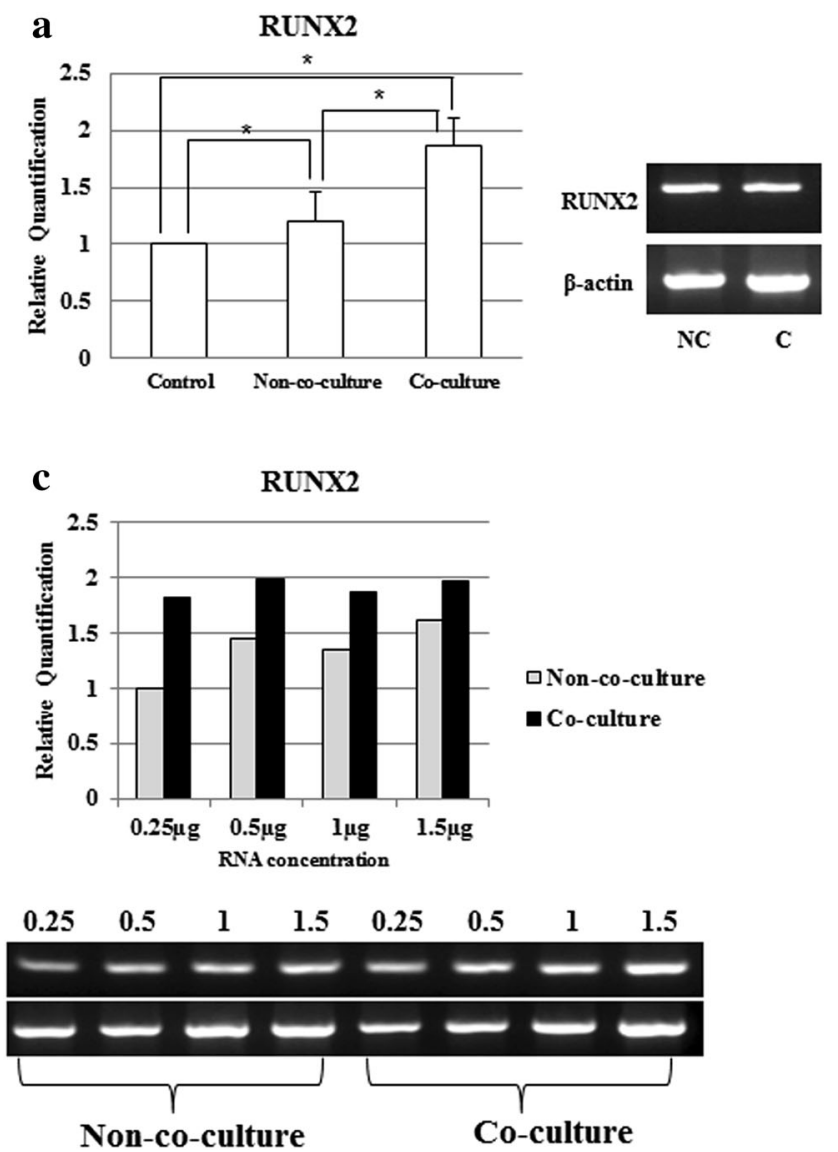

Fig. 5 RUNX2 and PPAR $\gamma 2$ gene expression comparison by quantitative RT-PCR. After co-cultured for 2 weeks, osteogenic differentiation medium was replaced for induction the osteogenesis. a, b Real-time PCR was performed for analyzing the RUNX2 and PPAR $\gamma 2$ gene expression after osteogenic induction in co-culture DFAT cells comparing to non-co-culture and undifferentiated DFAT cells. There was statistically significant difference in RUNX2 gene expression level in co-culture group with in contrast of PPAR $\gamma 2$ gene

signaling molecules, including MEK/ERK, p38 MAPK, and COX-2/PGE2/VEGF in hypoxia condition enhanced osteogenic differentiation. While all factors of the osteogenic lineage demonstrated higher in co-culture versus non-coculture groups, the effect of co-culture was not clearly identified, other than the hypoxia effects $[19,39]$.

Using adipose tissue as a cell-based procedure for periodontal regeneration has recently surfaced in the transplantation of ASCs in the oral rat model [23-25]. The role of DFAT cells in periodontal regeneration, however, was not investigated. Despite the presence of DFAT cells in the adipogenic lineage, they were enhanced by proper environment and they could be induced for osteogenic differentiation. This suggested that DFAT cells offered a potent function for differentiation. Therefore, DFAT cells could become a novel somatic cell source for periodontal regeneration that uses the co-culture system to enhance osteogenesis.
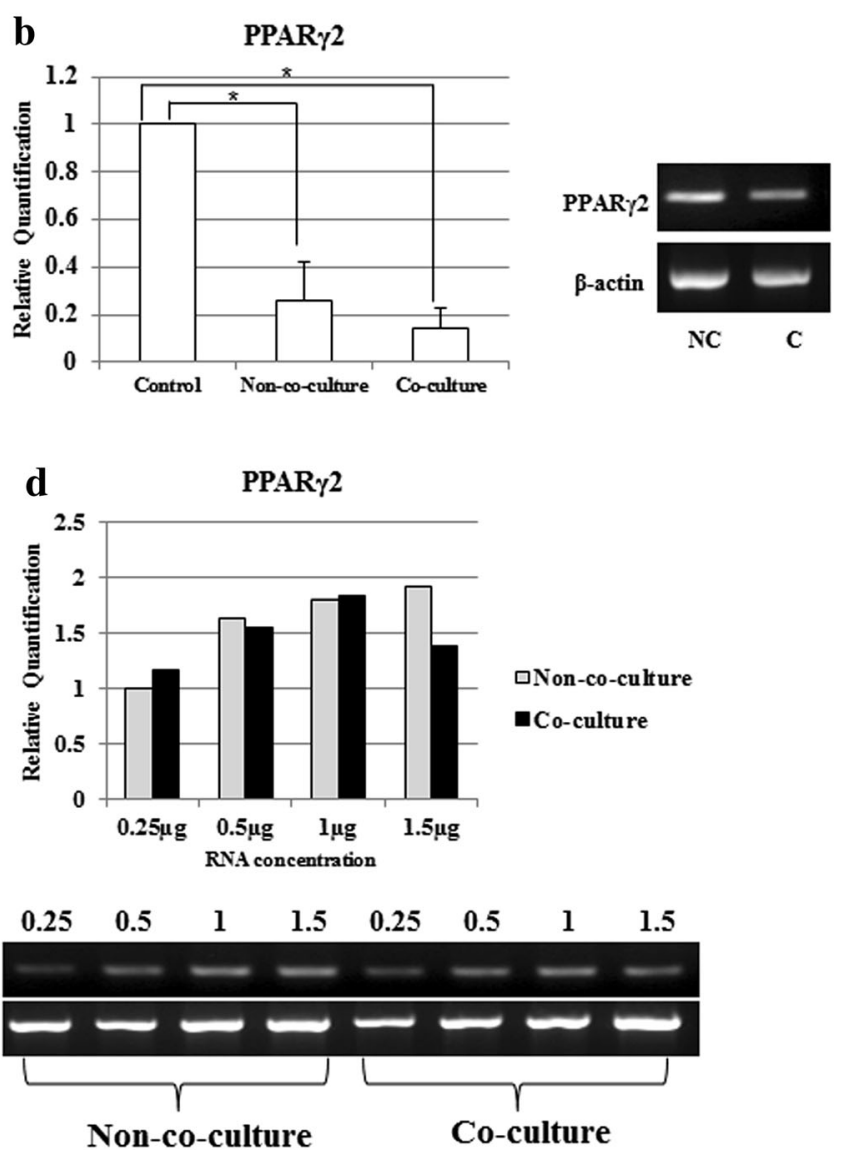

expression level. c, d Semi-quantitative RT-PCR was analyzed in serial of RNA concentration. RUNX2 gene expression has upregulated in all concentration of co-culture group. In contrast to PPAR $\gamma 2$, gene expression has down-regulated in co-culture group comparing to non-coculture group at 0.5 and $1.5 \mu \mathrm{g}$. $\mathrm{NC}$ means nonco-culture, $C$ means co-culture. Astrerisk indicates that comparison was statistically significant difference at $P<0.05$

\section{Conclusion}

Our finding first demonstrated the co-culture effect of DFAT cells with PDLSC in aspects of methylation profiles and in enhancing osteogenic gene expression. We also demonstrated the contrary effects between the osteogenic and adipogenic lineages through using the gene expression level. We concluded that DFAT cells might be an alternative cell-based therapy for periodontal regeneration.

Acknowledgments This work was funded by the Nippon Dental University School of Life Dentistry. I would like to thank Professor Taka Nakahara (Department of Development and Regenerative Dentistry, Nippon Dental University School of Life Dentistry at Tokyo) who provides knowledge and profitably suggestions. I thank Associate Professor Akihiro Oyama (Department of NDU Life Sciences, Nippon Dental University School of Life Dentistry at Tokyo) who coordinated for sample obtaining and laboratory techniques. 
Open Access This article is distributed under the terms of the Creative Commons Attribution License which permits any use, distribution, and reproduction in any medium, provided the original author(s) and the source are credited.

\section{References}

1. Melcher AH. On the repair potential of periodontal tissues. J Periodontol. 1976;47:256-60.

2. Benatti BB, Silvério KG, Casati MZ, Sallum EA, Nociti FH. Physiological features of periodontal regeneration and approaches for periodontal tissue engineering utilizing periodontal ligament cells. J Biosci Bioeng. 2007;103:1-6.

3. Bartold PM, Xiao Y, Lyngstaadas SP, Paine ML, Snead ML. Principles and applications of cell delivery systems for periodontal regeneration. Periodontology. 2000;2006(41):123-35.

4. Lin NH, Gronthos S, Bartold PM. Stem cells and periodontal regeneration. Aust Dent J. 2008;53:108-21.

5. Huang GT, Gronthos S, Shi S. Mesenchymal stem cells derived from dental tissues vs. those from other sources: their biology and role in regenerative medicine. J Dent Res. 2009;88:792-806.

6. Hynes K, Menicanin D, Gronthos S, Bartold PM. Clinical utility of stem cells for periodontal regeneration. Periodontology. 2000;2012(59):203-27.

7. Tamaki $Y$, Nakahara $T$, Ishikawa $H$, Sato $S$. In vitro analysis of mesenchymal stem cells derived from human teeth and bone marrow. Odontology. 2012;101:121-32.

8. Nagatomo K, Komaki M, Sekiya I, Sakaguchi Y, Noguchi K, Oda $\mathrm{S}$, et al. Stem cell properties of human periodontal ligament cells. J Periodontal Res. 2006;41:303-10.

9. Gay IC, Chen S, MacDougall M. Isolation and characterization of multipotent human periodontal ligament stem cells. Orthod Craniofac Res. 2007;10:149-60.

10. Liu L, Ling J, Wei X, Wu L, Xiao Y. Stem cell regulatory gene expression in human adult dental pulp and periodontal ligament cells undergoing odontogenic/osteogenic differentiation. J Endod. 2009;35:1368-76.

11. Seo B, Miura M, Gronthos S, Bartold P, Batouli S, Brahim J, et al. Investigation of multipotent postnatal stem cells from human periodontal ligament. Lancet. 2004;364:149-55.

12. Matsumoto T, Kano K, Kondo D, Fukuda N, Iribe Y, Tanaka N, et al. Mature adipocyte-derived dedifferentiated fat cells exhibit multilineage potential. J Cell Physiol. 2008;215:210-22.

13. Sugihara H, Yonemitsu N, Miyabara S, Yun K. Primary cultures of unilocular fat cells: characteristics of growth in vitro and changes in differentiation properties. Differentiation. 1986;31:42-9.

14. Sugihara H, Yonemitsu N, Miyabara S, Toda S. Proliferation of unilocular fat cells in the primary culture. J Lipid Res. 1987;28:1038-45.

15. Poloni A, Maurizi G, Leoni P, Serrani F, Mancini S, Frontini A, et al. Human dedifferentiated adipocytes show similar properties to bone marrow-derived mesenchymal stem cells. Stem Cells. 2012;30:965-74.

16. Jumabay M, Zhang R, Yao Y, Goldhaber JI, Boström KI. Spontaneously beating cardiomyocytes derived from white mature adipocytes. Cardiovasc Res. 2010;85:17-27.

17. Sakuma T, Matsumoto T, Kano K, Fukuda N, Obinata D, Yamaguchi K, et al. Mature, adipocyte derived, dedifferentiated fat cells can differentiate into smooth muscle-like cells and contribute to bladder tissue regeneration. J Urol. 2009;182:355-65.

18. Obinata D, Matsumoto T, Ikado Y, Sakuma T, Kano K, Fukuda $\mathrm{N}$, et al. Transplantation of mature adipocyte-derived dedifferentiated fat (DFAT) cells improves urethral sphincter contractility in a rat model. Int J Urol. 2011;18:827-34.

19. Zhao L, Wu Y, Tan L, Xu Z, Wang J, Zhao Z, et al. Coculture with endothelial cells enhances osteogenic differentiation of periodontal ligament stem cells via cyclooxygenase-2/prostaglandin E2/vascular endothelial growth factor signaling under hypoxia. J Periodontol. 2013;84:1847-57.

20. Aoki S, Toda S, Sakemi T, Sugihara H. Coculture of endothelial cells and mature adipocytes actively promotes immature preadipocyte development in vitro. Cell Struct Funct. 2003;28:55-60.

21. Proksch S, Steinberg T, Stampf S, Schwarz U, Hellwig E, Tomakidi P. Crosstalk on cell behavior in interactive cocultures of hMSCs with various oral cell types. Tissue Eng Part A. 2012;18:2601-10.

22. Hong JH, Hwang ES, McManus MT, Amsterdam A, Tian Y, Kalmukova R, et al. TAZ, a transcriptional modulator of mesenchymal stem cell differentiation. Science. 2005;309:1074-8.

23. Tobita M, Uysal AC, Ogawa R, Hyakusoku H, Mizuno H. Periodontal tissue regeneration with adipose-derived stem cells. Tissue Eng Part A. 2008;14:945-53.

24. Tobita M, Mizuno H. Periodontal disease and periodontal tissue regeneration. Curr Stem Cell Res Ther. 2010;5:168-74.

25. Tobita M, Mizuno H. Adipose-derived stem cells for periodontal tissue regeneration. Methods Mol Biol. 2011;702:461-70.

26. Bernstein BE, Meissner A, Lander ES. The mammalian epigenome. Cell. 2007;128:669-81.

27. Boquest A, Shahdadfar A. Isolation and transcription profiling of purified uncultured human stromal stem cells: alteration of gene expression after in vitro cell culture. Mol Biol Cell. 2005;16:1131-41.

28. Teven CM, Liu X, Hu N, Tang N, Kim SH, Huang E, et al. Epigenetic regulation of mesenchymal stem cells: a focus on osteogenic and adipogenic differentiation. Stem Cells Int. 2011;2011:201371.

29. Kanai F, Marignani PA, Sarbassova D, Yagi R, Hall RA, Donowitz M, et al. TAZ: a novel transcriptional co-activator regulated by interactions with 14-3-3 and PDZ domain proteins. EMBO J. 2000;19:6778-91.

30. Cui CB, Cooper LF, Yang X, Karsenty G, Aukhil I. Transcriptional coactivation of bone-specific transcription factor $\mathrm{Cbfa} 1$ by TAZ. Mol Cell Biol. 2003;23:1004-13.

31. Murakami M, Nakagawa M, Olson EN, Nakagawa O. A WW domain protein TAZ is a critical coactivator for TBX5, a transcription factor implicated in Holt-Oram syndrome. Proc Natl Acad Sci USA. 2005;102:18034-9.

32. Jeon MJ, Kim JA, Kwon SH, Kim SW, Park KS, Park S-W, et al. Activation of peroxisome proliferator-activated receptor-gamma inhibits the Runx2-mediated transcription of osteocalcin in osteoblasts. J Biol Chem. 2003;278:23270-7.

33. Reid IR. Relationships between fat and bone. Osteoporos Int. 2008;19:595-606.

34. Zaidi M, Buettner C, Sun L, Iqbal J. Minireview: the link between fat and bone: does mass beget mass? Endocrinology. 2012;153:2070-5.

35. Harada S, Rodan GA. Control of osteoblast function and regulation of bone mass. Nature. 2003;423:349-55.

36. Ducy P, Zhang R, Geoffroy V, Ridall AL, Karsenty G. Osf2/ Cbfa1: a transcriptional activator of osteoblast differentiation. Cell. 1997;89:747-54.

37. Lian JB, Stein GS, Stein JL, van Wijnen AJ. Osteocalcin gene promoter: unlocking the secrets for regulation of osteoblast growth and differentiation. J Cell Biochem Suppl. 1998;30-31:62-72.

38. Takano M, Otsuka F, Matsumoto Y, Inagaki K, Takeda M, Nakamura E, et al. Peroxisome proliferator-activated receptor activity is involved in the osteoblastic differentiation regulated by bone morphogenetic proteins and tumor necrosis factor- $\alpha$. Mol Cell Endocrinol. 2012;348:224-32.

39. Wu Y, Cao H, Yang Y, Zhou Y, Gu Y, Zhao X, et al. Effects of vascular endothelial cells on osteogenic differentiation of noncontact co-cultured periodontal ligament stem cells under hypoxia. J Periodontal Res. 2013;48:52-65.

40. Population doubling time, http://www.doubling-time.com/com pute.php. 